\title{
Contact Line Instability and Pattern Selection in Thermally Driven Liquid Films
}

\author{
Roman O. Grigoriev \\ School of Physics, Georgia Institute of Technology, Atlanta, GA 30332-0430
}

(October 25, 2018)

\begin{abstract}
Liquids spreading over a solid substrate under the action of various forces are known to exhibit a long wavelength contact line instability. We use an example of thermally driven spreading on a horizontal surface to study how the stability of the flow can be altered, or patterns selected, using feedback control. We show that thermal perturbations of certain spatial structure imposed behind the contact line and proportional to the deviation of the contact line from its mean position can completely suppress the instability. Due to the presence of mean flow and a spatially nonuniform nature of spreading liquid films the dynamics of disturbances is governed by a nonnormal evolution operator, opening up a possibility of transient amplification and nonlinear instabilities. We show that in the case of thermal driving the nonnormality can be significant, especially for small wavenumber disturbances, and trace the origin of transient amplification to a close alignment of a large group of eigenfunctions of the evolution operator. However, for values of noise likely to occur in experiments we find that the transient amplification is not sufficiently strong to either change the predictions of the linear stability analysis or invalidate the proposed control approach.
\end{abstract}

\section{INTRODUCTION}

Driven spreading of liquid films is a process which occurs in numerous industrial coating applications, such as spin-coating of hard drives, so understanding its dynamics and learning to control it is very important. For instance, instabilities which arise during the spreading of the liquid on the solid substrate can lead to nonuniform coverage, adversely affecting the quality of produced coating. Driven spreading of thin films and patterning also have important implications for microfluidics.

Driven spreading of liquid films under the action of gravity $^{1,2}$, centrifugal acceleration ${ }^{3}$, thermocapillary effects $^{4}$, or combination thereof ${ }^{5}$ has been extensively studied in the literature. Stability analysis of such flows has attracted the most attention and the mechanism of the linear instability is now well understood. Considerable progress has also been reached in feedback control of flat liquid layers ${ }^{6-8}$, whose dynamics is governed by normal differential operators. The attempts to influence the stability of spreading films have so far been limited to non-feedback control achieved through either imposing an externally generated counterflow ${ }^{9}$ or chemically patterning the substrate ${ }^{10,11}$.

This study represents the first theoretical treatment of the feedback control problem for spreading films. The spatially nonuniform nature of spreading films and the presence of mean flow make the control problem much more difficult compared to the case of flat stationary films, because the dynamics of disturbances in spreading films is governed by a nonnormal evolution operator and thus requires a more careful analysis. For instance, liquid films flowing down an incline have been found to develop a contact line instability when the linear analysis predicted stable evolution ${ }^{12}$. We derive the slip model of thermally driven spreading and use it to show that the contact line instability can be suppressed using adaptive thermal perturbations which depend on the distortion of the contact line. (This type of feedback is chosen because it is easiest to implement experimentally with sufficient spatial and temporal resolution via optical means ${ }^{13}$.) $\mathrm{Al}-$ though the results of the following analysis should be applicable regardless of the driving force, we concentrate our attention on the case of thermal driving.

The layout of the paper is as follows. The slip model of thermally driven liquid films is derived in Section II and its linear stability analysis is conducted in Section III. The validity of linear stability analysis and transient effects are considered in Section IV. Section V presents the proposed algorithm for feedback control of the contact line instability and Section VI contains the conclusions.

\section{SLIP MODEL FOR THERMAL SPREADING}

We consider the spreading of a thin layer of partially wetting liquid on a horizontal substrate (see Fig. 1). The spreading process is conventionally described using the lubrication approximation ${ }^{14}$, with the horizontal velocity governed by the Stokes equation

$$
\mu \partial_{z z} \mathbf{v}=\nabla \bar{p},
$$

where $\mu$ is the dynamic viscosity, $\bar{p}$ is the modified pressure, $\nabla=\left(\partial_{x}, \partial_{y}\right)$ is the 2 -dimensional gradient operator, and the vertical velocity is neglected.

It is well known ${ }^{14,15}$ that the standard no-slip boundary condition at the liquid-solid interface results in a stress singularity at the contact line. The only approach explored in the literature for the thermally driven case 


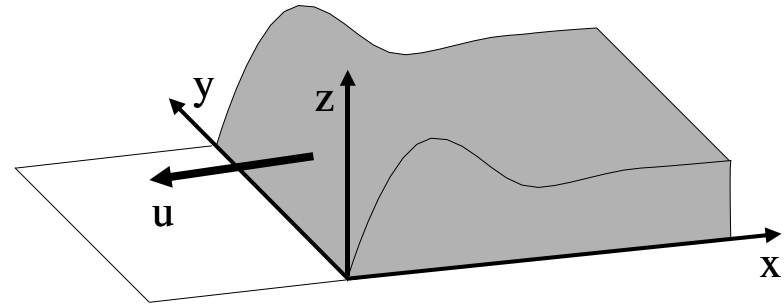

FIG. 1. Spreading liquid film on a solid substrate.

was to relieve this singularity by introducing a thin precursor film ${ }^{5}$. However, as we intend to use the position of the contact line in our control algorithm later, the precursor model becomes inconvenient. Instead we choose to specify a microscopic contact angle and relieve the stress singularity by employing a partial slip boundary condition

$$
\mathbf{v}=\frac{\alpha}{3 h} \partial_{z} \mathbf{v}
$$

at the bottom of the liquid layer, where $h$ is the local thickness of the film, and $\alpha$ is the phenomenological slip coefficient. The slip boundary condition (2) was originally introduced by Greenspan ${ }^{16}$ for modeling the unforced spreading of liquid drops and later used to model the forced spreading of liquid films under the action of gravity $^{17}$. At the free surface the standard stress balance boundary condition

$$
\mu \partial_{z} \mathbf{v}=\nabla \sigma
$$

applies, where $\sigma$ is the surface tension coefficient. Solving (1) subject to these boundary conditions we obtain the horizontal velocity

$$
\mathbf{v}=\frac{1}{\mu} z \nabla \sigma-\frac{1}{\mu}\left(\frac{\alpha}{3}+h z-\frac{1}{2} z^{2}\right) \nabla \bar{p} .
$$

In order to make the phenomenological boundary condition (2) consistent with the physics of the flow, in (4) we have dropped an unphysical term $\alpha \nabla \sigma / 3 \mu h$ which diverges for vanishing film thickness. This divergence is not necessarily a cause for alarm as the continuous approximation underlying the Stokes equation itself breaks down in this limit. The shear stress also becomes poorly defined for very thin films. Our choice of the functional form for the horizontal velocity (4), therefore, amounts to picking an appropriate phenomenological model in the region where the continuous description of the flow becomes invalid and fluctuations become important.

For a film which is sufficiently thin the hydrostatic pressure can be ignored, so that the modified pressure is given by the normal component of the surface tension $\bar{p}=-\sigma \kappa=-\sigma \nabla^{2} h$. Substituting (4) into the mass conservation condition

$$
\partial_{t} h=-\int_{0}^{h}(\nabla \cdot \mathbf{v}) d z
$$

and integrating we obtain an evolution equation for the thickness:

$$
\partial_{t} h=-\nabla \cdot\left[\frac{1}{2 \mu} h^{2} \nabla \sigma+\frac{1}{3 \mu}\left(\alpha h+h^{3}\right) \nabla\left(\sigma \nabla^{2} h\right)\right] .
$$

Now consider the situation which arises when the substrate covered by the liquid film is subjected to a linear temperature gradient in the $x$-direction. Assuming that the surface tension changes linearly with temperature $\theta$,

$$
\sigma(x)=\sigma\left(\theta_{0}\right)+x \partial_{x} \theta \partial_{\theta} \sigma \equiv \sigma_{0}-\tau x,
$$

and neglecting the variation in $\sigma$ in the second term of (6), which produces subdominant contribution (see, e.g., the discussion in Ref. 5), we obtain

$$
\begin{aligned}
\partial_{t} h=\frac{\tau}{2 \mu} \partial_{x} h^{2} & -\frac{\sigma_{0}}{3 \mu} \partial_{x}\left[\left(\alpha h+h^{3}\right)\left(\partial_{x x x} h+\partial_{x y y} h\right)\right] \\
& -\frac{\sigma_{0}}{3 \mu} \partial_{y}\left[\left(\alpha h+h^{3}\right)\left(\partial_{x x y} h+\partial_{y y y} h\right)\right] .
\end{aligned}
$$

We can absorb most parameters into the spatial and temporal scales by introducing the nondimensional variables $t^{\prime}=t / T, x^{\prime}=x / X, y^{\prime}=y / X$, and $h^{\prime}=h / H$. The vertical length scale $H$ is defined by the characteristic thickness of the film and sets both the horizontal length scale $X=\left[2 \sigma_{0} H^{2} / 3 \tau\right]^{1 / 3}$ and the time scale $T=2 \mu X / \tau H$. After defining the dimensionless slip coefficient $\alpha^{\prime}=\alpha / H^{2}$ and dropping the primes we obtain

$$
\begin{aligned}
\partial_{t} h=\partial_{x} h^{2} & -\partial_{x}\left[\left(\alpha h+h^{3}\right)\left(\partial_{x x x} h+\partial_{x y y} h\right)\right] \\
& -\partial_{y}\left[\left(\alpha h+h^{3}\right)\left(\partial_{x x y} h+\partial_{y y y} h\right)\right] .
\end{aligned}
$$

The obtained equation has the same form as the one describing gravity driven rather than temperature driven films (see, e.g., equation (33) in Ref. 17), with the exception that $h^{2}$ in the first term on the right-hand-side is replaced with $\alpha h+h^{3}$. This similarity of the structure of the governing equations suggests that the feedback control problem can be treated in the same way regardless of the nature of the driving force.

The liquid spreads in the direction opposite to the temperature gradient, so the motion of the contact line is most conveniently described in the reference frame moving with speed $u$ towards negative $x$. In this frame the evolution equation possesses a transversely uniform steady state solution, which gives the asymptotic film profile for constant flux boundary conditions. Substituting $h(x, y, t)=h_{0}(x+u t)$ into (9) and integrating once we obtain

$$
u h_{0}-h_{0}^{2}+\left(\alpha h_{0}+h_{0}^{3}\right) h_{0}^{\prime \prime \prime}=d
$$

where prime indicates the differentiation with respect to the $x$ coordinate. The constants $u$ and $d$ can be determined from the appropriate boundary conditions. Following Spaid and Homsy ${ }^{17}$ we require that the film thickness vanish at the contact line, $h_{0}(0)=0$, and specify the slope $h_{0}^{\prime}(0)=c \equiv(X / H) \tan \phi$, where $\phi$ is the 


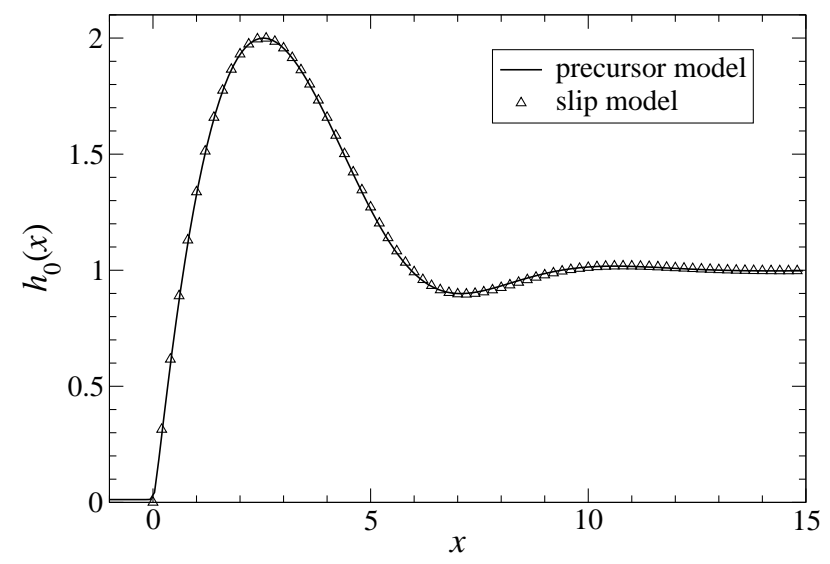

FIG. 2. Asymptotic film profiles produced by the precursor model (12) with $b=0.0119$ and the slip model with $\alpha=0.1$ and $c=1.53$. The parameters were chosen to produce a capillary ridge of height $h_{\max }=2$.

microscopic dynamic contact angle in the dimensional variables. Furthermore, the constant flux boundary condition at the tail of the film far away from the contact line gives $h_{0}(\infty)=1$ (this choice corresponds to using the dimensional tail thickness as the vertical lengthscale, $H)$ and $h_{0}^{\prime \prime \prime}(\infty)=0$. These boundary conditions require $d=0$ and $u=1$, and consequently

$$
h_{0}^{\prime \prime \prime}=\frac{h_{0}-1}{h_{0}^{2}+\alpha} .
$$

The solution to this equation describes the height profile of the spreading film once the distance from the contact line to the reservoir becomes sufficiently large.

At this point it is appropriate to make two comments regarding the structure of the lubrication equations produced by the slip model. First of all, from a mathematical perspective, introduction of partial slip at the liquid-solid interface is equivalent to a regularization procedure for a singular differential equation. Dropping the diverging term in (4) is equivalent to keeping the regularization parameter $\alpha$ only in the terms in (6) responsible for the singular behavior of the solution at the contact line. We could have kept all the terms in (4) and (6) just as well. Neither (9) nor (11) would have changed.

Second, (11) is very similar to the equation produced by the precursor model of Kataoka and Troian ${ }^{5}$

$$
h_{0}^{\prime \prime \prime}=\frac{\left(h_{0}-1\right)\left(h_{0}-b\right)}{h_{0}^{3}},
$$

where $b$ is the thickness of the precursor layer. In fact, the solutions of the two equations are virtually indistinguishable (see Fig. 2) for the proper choice of parameters (precursor thickness, slip coefficient, and contact angle). This is hardly a surprise, as the two equations become identical in the limit $b \rightarrow 0$ and $\alpha \rightarrow 0$, and proves that the final result is essentially independent of the regularization procedure used to get rid of the stress singularity at the contact line.

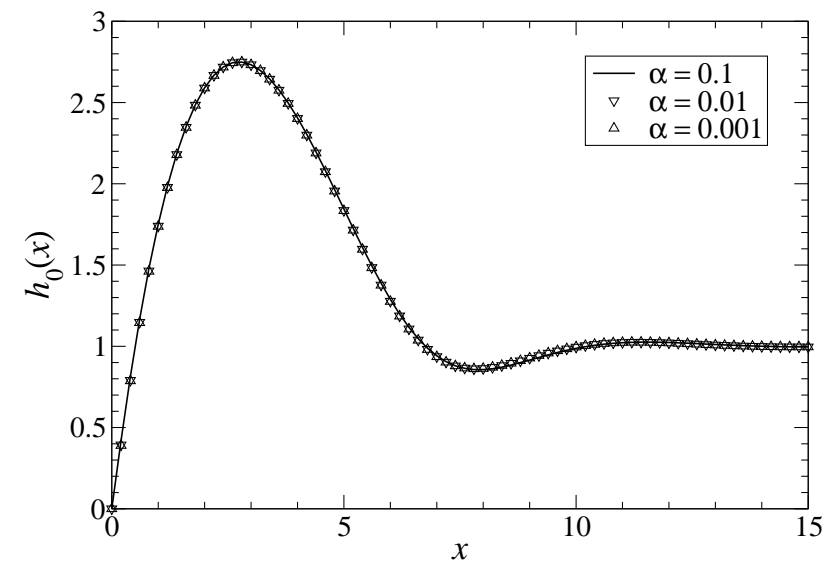

FIG. 3. Asymptotic film profiles with a fixed height of the capillary ridge $h_{\max }=2.75$ and different values of the slip coefficient.

The slip model has two independent parameters $(c$ and $\alpha$ ), while the precursor model has only one (b). A closer inspection shows that the gross features of the slip model are, in fact, determined by a single parameter (say, the height of the capillary ridge). As Fig. 3 shows, one can change the value of the slip coefficient by two orders of magnitude without causing noticeable changes in the film profile (the contact angle will also change in response to the changes in $\alpha$ to keep the height of the capillary ridge constant). The second parameter fine tunes the shape of the film in the immediate vicinity of the contact line. The slip model is, therefore, not only more convenient for the purpose of control (it explicitly defines the easy to measure position of the contact line), but it is to some extent more flexible than the precursor model.

Finally, let us explore the dependence of the film profile on the parameters. As we have just seen, the profile is rather insensitive to the changes in the slip coefficient, so let us fix it and vary the contact angle (see Fig. 4). The first important observation is that regardless of the magnitude of the contact angle, the capillary ridge never disappears (this turns out to be the case for any reasonable value of the slip coefficient, i.e., $\alpha<1$ ). This is in contrast with the results obtained for gravity driven films, where the capillary ridge completely disappears at small inclination angles ${ }^{12}$. Second, the height of the ridge is a monotonically increasing function of $c$ and becomes essentially independent of the contact angle for $c<0.3$. The dependence on the slip coefficient is more subtle: the value of $\alpha$ controls the minimum height of the capillary ridge which grows with decreasing slip coefficient.

\section{CONTACT LINE INSTABILITY}

Linear stability of the asymptotic solution $h_{0}(x+u t)$ can be determined in a standard way ${ }^{2,5,12}$. Since this solution is uniform in the transverse direction, the linearized equation describing the dynamics of small dis- 


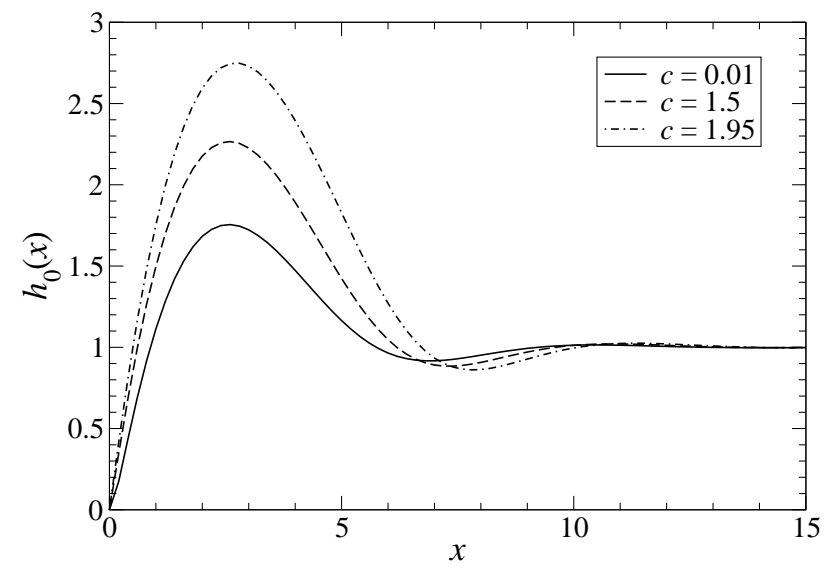

FIG. 4. Asymptotic film profiles for different values of the contact angle. The slip coefficient is held fixed at $\alpha=0.01$.

turbances can be partially diagonalized by Fourier transforming it in the $y$-direction. By substituting

$$
h(x, y, t)=h_{0}(x+u t)+\epsilon g(x+u t, t) e^{i q y}
$$

into (9) and retaining terms of order $\epsilon$, we obtain

$$
\partial_{t} g=L(q) g
$$

where $L(q)=L_{0}+q^{2} L_{1}+q^{4} L_{2}$ is a $4^{\text {th }}$ order differential operator defined via

$$
\begin{aligned}
& L_{0} g=-\left[\left\{1-2 h_{0}+\left(\alpha+3 h_{0}^{2}\right) h_{0}^{\prime \prime \prime}\right\} g+\left(\alpha h_{0}+h_{0}^{3}\right) g^{\prime \prime \prime}\right]^{\prime}, \\
& L_{1} g=\left[\left(\alpha+3 h_{0}^{2}\right) h_{0}^{\prime} g\right]^{\prime}+2\left(\alpha h_{0}+h_{0}^{3}\right) g^{\prime \prime} \\
& L_{2} g=-\left(\alpha h_{0}+h_{0}^{3}\right) g .
\end{aligned}
$$

The boundary condition on $g$ at the contact line

$$
h_{0}^{\prime \prime}(0) g(0)=h_{0}^{\prime}(0) g^{\prime}(0)
$$

can be obtained by requiring the perturbed solution to have the same contact angle as the unperturbed solution. The other three boundary conditions can all be imposed at the tail, say,

$$
g(\infty)=g^{\prime}(\infty)=g^{\prime \prime}(\infty)=0 .
$$

Even though we cannot find the eigenvalues of $L(q)$ for arbitrary $q$ analytically, for long wavelength disturbances we can use perturbation theory to get the leading order (in $q^{2}$ ) terms. This requires finding the eigenfunctions of $L_{0}$ and its adjoint, $L_{0}^{\dagger}$. Taking the second derivative of (10) we obtain

$$
L_{0} h_{0}^{\prime}=0
$$

so that $g_{0}=h_{0}^{\prime}$ is an eigenfunction of $L_{0}$ with eigenvalue $\beta_{0}^{0}=0$. The adjoint operator is defined via

$$
\begin{aligned}
L_{0}^{\dagger} f & =\left[1-2 h_{0}+\left(\alpha+3 h_{0}^{2}\right) h_{0}^{\prime \prime \prime}\right] f^{\prime} \\
& -\left[\left(\alpha h_{0}+h_{0}^{3}\right) f^{\prime}\right]^{\prime \prime \prime},
\end{aligned}
$$

so its respective eigenfunction is just a constant, say, $f_{0}=1$. In fact, these are generic results with deep physical meaning. Identical relations between the asymptotic state and the leading eigenfunctions were obtained, e.g., for gravity driven films using the precursor model ${ }^{12,18}$. The relation for $g_{0}$ is due to the fact that equations for the asymptotic state are translationally invariant in the direction of the flow (this reflects an arbitrary choice in the position of the contact line), while the relation for $f_{0}$ is the consequence of the divergence form of (5), which reflects mass conservation.

According to the perturbation theory the leading eigenvalue has the following $q$-dependence:

$$
\beta_{0}(q)=\beta_{0}^{0}+q^{2} \frac{\int_{0}^{\infty} f_{0}^{*} L_{1} g_{0} d x}{\int_{0}^{\infty} f_{0}^{*} g_{0} d x}+O\left(q^{4}\right) .
$$

Using (11) this can be reduced to

$$
\beta_{0}(q)=q^{2} \int_{0}^{\infty} h_{0}\left(h_{0}-1\right) d x+O\left(q^{4}\right) .
$$

As this is the largest eigenvalue, its sign determines the stability of the asymptotic state. It is easy to see that, if the asymptotic profile were monotonic, $0<h_{0}<1$, the integral would be strictly negative and the system would be linearly stable with respect to long wavelength disturbances. However, the presence of a capillary ridge makes the integral positive, showing that the increased mobility of the ridge provides the mechanism for the long wavelength instability in the thermally driven case. This mechanism has been originally conjectured by Kataoka and Troian based on the energy analysis of the precursor model $^{5}$. Equation (21) gives an explicit condition on the shape of the capillary ridge which determines the onset of instability, and echoes a similar result obtained for the case of gravity-driven flows ${ }^{2,12}$.

Substituting $g(x, t)=h_{0}^{\prime}(x) \exp \left[\beta_{0}(q) t\right]$ into (13) we notice that for small disturbances the right hand side represents the first two terms of the Taylor expansion of a distorted asymptotic state $h_{0}(x+\xi+u t)$, where

$$
\xi(y, t)=\epsilon e^{i q y+\beta_{0}(q) t} .
$$

is the deviation of the contact line from the mean. In fact, the marginal translational mode $g_{0}$ is not the only eigenfunction of $L_{0}$. There is an infinite discrete spectrum of eigenvalues $\beta_{n}$ and eigenfunctions $g_{n}$. Therefore, in the presence of an arbitrary disturbance (22) should be replaced with

$$
\xi(y, t)=\frac{1}{c} \sum_{n} g_{n}(0) \int_{-\infty}^{\infty} \epsilon_{n}(q) e^{i q y+\beta_{n}(q) t} d q .
$$

As the asymptotic state is unstable, the amplitude of the distortion will grow exponentially in time and eventually the contact line will form equally spaced "fingers". In order to calculate the wavenumber of the pattern we 
numerically compute the eigenfunctions and eigenvalues of the evolution operator $L(q)$. This is most easily accomplished by discretizing the eigenvalue equation on a spatially nonuniform mesh to properly resolve the rapid change in the solutions near the contact line. As both the asymptotic state $h_{0}$ and the eigenfunctions $g_{n}$ exponentially flatten for large $x$, a truncated domain can be used, so that the boundary conditions (17) are imposed at finite distance $l_{x}$ away from the contact line (we used $l_{x}=80$ in most of the calculations). In order to compute the asymptotic state we used a shooting method on a nonuniform mesh with roughly $10^{4}$ points. As $h=1$ is an unstable fixed point of (11), numerical integration (we used the $4^{\text {th }}$ order Runge-Kutta method) cannot be performed beyond $l_{x} \approx 30$ using double precision arithmetics. To determine the solution for a longer interval we used a method ${ }^{19}$ in which the numerical solution is extended using the analytical solution of (11) for $h \approx 1$ :

$$
h(x)=1+[a \cos (\sqrt{3} \kappa x)+b \sin (\sqrt{3} \kappa x)] e^{-\kappa x}+\cdots,
$$

where $\kappa=(1+\alpha)^{-1 / 3} / 2$ and $a, b$ are constants determined by a least squares fit. The eigenvalues and eigenfunctions where then computed using a built-in function in Matlab on a 1025-point mesh (finer resolutions did not change the eigenvalues by more than about $5 \%$ ).

The results of our calculations for a typical choice of parameters are presented in Fig. 5. The fastest growing disturbance is found to have a transverse wavenumber $q_{\max }$ lying between 0.29 and 0.34 . This wavenumber decreases with the thickness of the capillary ridge $h_{\max }$ and gives the characteristic wavelength of the fingering pattern $\lambda_{\max }=2 \pi / q_{\max }$, which ranges between 18.5 and 21.7, in excellent agreement with the predictions of both the precursor model ${ }^{5}$ and the experiments ${ }^{4,20}$ which found the dimensionless wavelength to be between 18 and 22. The growth rate of the most unstable mode increases with $h_{\max }$, varying between 0.12 and 0.17 for the range of contact angles considered here. Brzoska et $a l .{ }^{20}$ have obtained an experimental value of about 0.15 , which is also consistent with the theory. As the thickness of the ridge has not been determined in experiments, it is impossible to make a more direct comparison, but nevertheless these results can be used to establish the ranges of parameters relevant for experimental conditions.

\section{LINEAR STABILITY AND TRANSIENT DYNAMICS}

We have determined earlier that the capillary ridge is present for any reasonable choice of parameters, so the asymptotic state of a thermally driven film is always linearly unstable and the contact line instability will inevitably set in as the asymptotic state is approached. However, as a quick comparison of (15) and (19) shows, the evolution operator $L(q)$ is nonnormal, and we have to
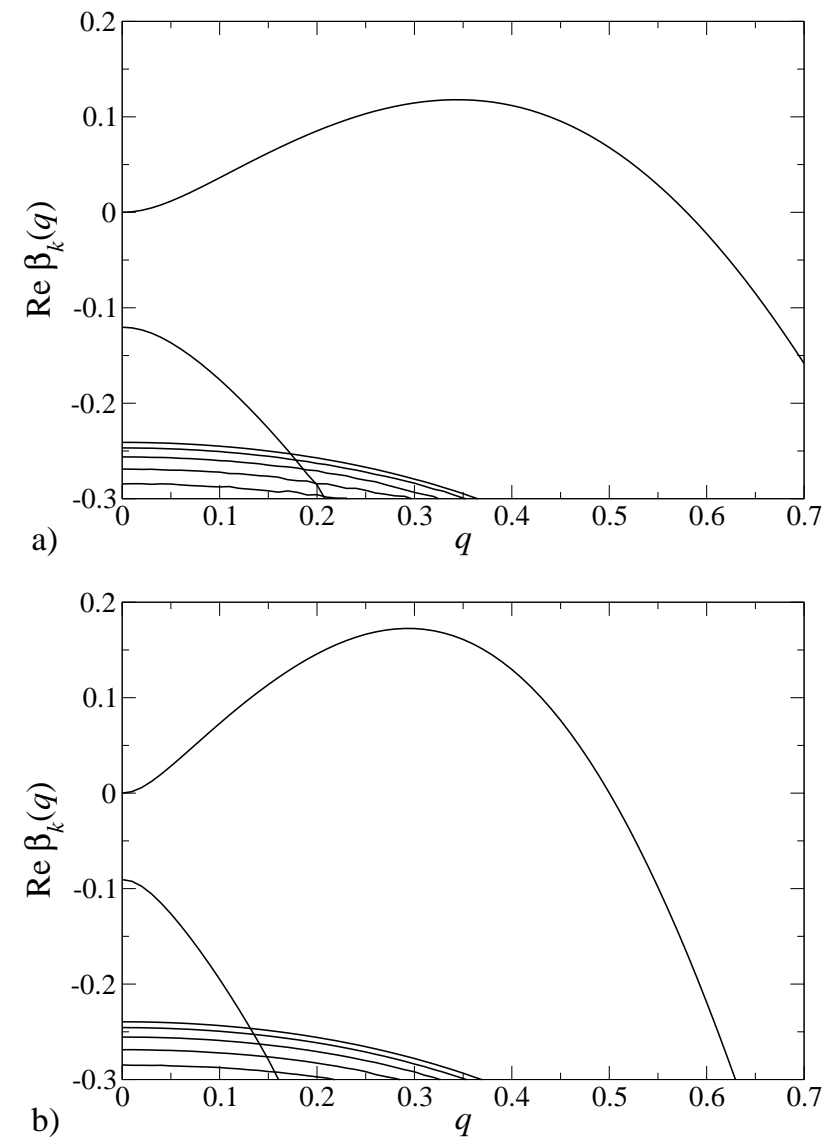

FIG. 5. The twelve leading eigenvalues of $L(q)$ for $\alpha=0.01$ and (a) $c=1\left(h_{\max }=1.93\right),(\mathrm{b}) c=1.95\left(h_{\max }=2.75\right)$. In both cases $\beta_{0}(0)$ and $\beta_{1}(0)$ are real, while the other ten eigenvalues come in complex conjugate pairs.

consider the possibility that the transient dynamics associated with nonnormality could change the predictions of the linear stability theory concerning the growth rates of disturbances. In fact, transient effects could be quite significant. For instance, turbulence in channel flows arises at values of the Reynolds number well below the critical one predicted by the linear stability analysis ${ }^{21}$. Both in the driven films and in channel flows nonnormality arises due to a significant mean flow, so it is natural to expect that transient behavior could be important for driven liquid films as well. Indeed, a disagreement between theoretical and experimental predictions of the most unstable wavelength in gravity driven films at low angles of inclination has been attributed to transient dynamics ${ }^{12}$.

Linear stability analysis presented in the previous section is based on the assumption that the nonlinear terms are negligible at all times. If the disturbances were small and their dynamics were governed by a normal evolution operator, this assumption would have been well justified. For instance, when the system is stable, the eigenvalues predict both the short term and the long term dynamics. The situation can change dramatically when the evolution operator becomes nonnormal, as the eigen- 


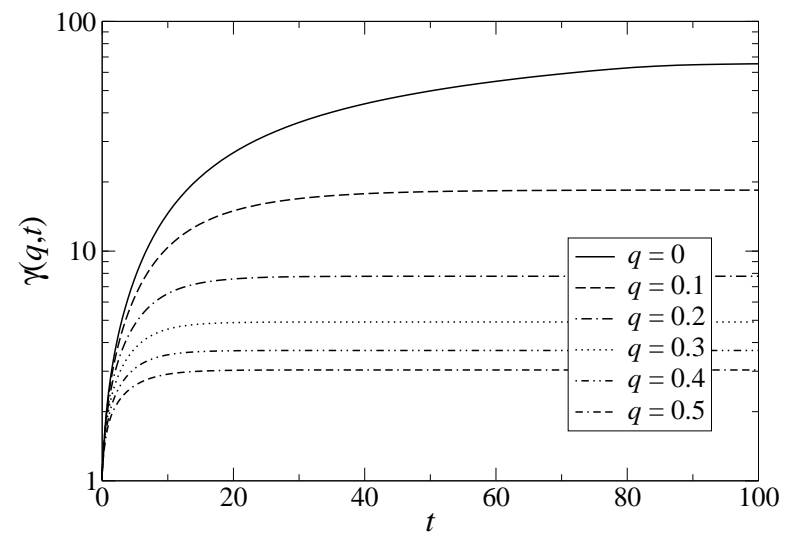

FIG. 6. Transient amplification $\gamma(q, t)$ as a function of time for $\alpha=0.01, c=1$ and different values of the wavenumber.

values become poor predictors of the short term dynamics. Inclusion of the nonlinear terms in (14) has two major consequences. First of all, nonlinear terms couple the dynamics of modes with different transverse wavenumbers ${ }^{18}$. Second, nonlinear terms produce deviations from the asymptotic state which can be transiently amplified due to the nonnormality of the linearized evolution operator ${ }^{21}$. A combination of these two effects can lead to a nonlinear instability which can compete with the linear instability.

There are two scenarios which could invalidate the results of linear stability analysis. In the simplest (purely linear) scenario ${ }^{12,22}$, an initial disturbance with transverse wavenumber $q_{0}$ and magnitude $\|g(x, 0)\|=\xi$ could be transiently amplified by a factor $\gamma\left(q_{0}\right)$ to produce a disturbance with magnitude $\gamma \xi=O(1)$. If this amplification occurs on a time scale shorter than $1 / \beta_{0}\left(q_{\max }\right)$ and $\xi \gtrsim \gamma^{-1}$, the transient effects will dominate and a distortion of the contact line with the wavenumber $q_{0}$ rather than $q_{\max }$ will result.

In a more complicated scenario ${ }^{21,23}$, an initial disturbance is transiently amplified by the linear part of the evolution operator, while the nonlinear terms produce secondary disturbances which are further transiently amplified. This could lead to a positive feedback loop bootstrapping a nonlinear instability, provided the secondary disturbances contain the wavenumber of the initial disturbance and have the magnitude which is at least as large. It is easy to check that the nonlinear evolution operator will only contain terms quadratic, cubic, and quartic in $g(x, t)$ in addition to the linear terms kept in (14). Only the cubic terms will contain the original wavenumber, so the secondary disturbances produced by the quadratic and quartic terms will not be further transiently amplified. (Initial disturbances with $q_{0}=0$ represent the only exception, but they do not lead to distortion of the contact line.) An initial disturbance of magnitude $\xi$, which is transiently amplified by a factor $\gamma$, will produce a secondary disturbance of magnitude $O\left((\gamma \xi)^{3}\right)$ via the cubic nonlinearities. The secondary disturbance will

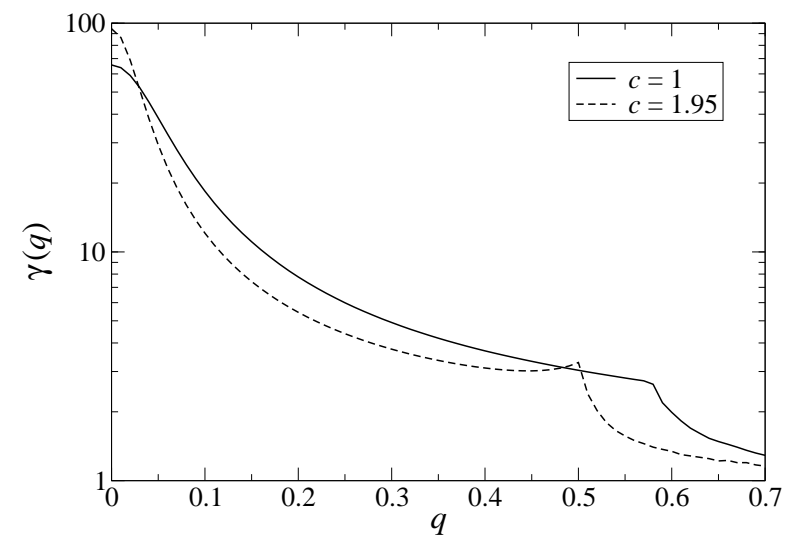

FIG. 7. Dependence of the maximal transient amplification $\gamma(q)$ on the wavenumber for $\alpha=0.01$ and different values of the contact angle.

exceed the primary disturbance only when $(\gamma \xi)^{3} \gtrsim \xi$, so that a self-sustaining nonlinear instability becomes possible for $\xi \gtrsim \gamma^{-3 / 2}$.

The maximal transient amplification in the stable band can be defined in a conventional manner ${ }^{23}$ :

$$
\gamma(q, t)=\sup _{g(x, 0)} \frac{\|g(x, t)\|}{\|g(x, 0)\|}=\left\|e^{L t}\right\| .
$$

In the unstable band the fast transient amplification will be followed by the slower exponential growth. Factoring out the exponential growth one obtains the following upper bound:

$$
\gamma(q, t)=\sup _{g(x, 0)} \frac{\|g(x, t)\|}{\|g(x, 0)\|} e^{-\beta_{0} t}=\left\|e^{\left(L-\beta_{0}\right) t}\right\| .
$$

Numerical calculations show that in the unstable band $\gamma(q, t)$ is a monotonically increasing function of time, so the maximum is reached for $t \rightarrow \infty$. The time dependence for several values of the transverse wavenumber is shown in Fig. 6. As $L-\beta_{0}$ and $L$ have the same eigenfunctions, we can easily calculate the matrix elements of the operator $U(t)=\exp \left[\left(L-\beta_{0}\right) t\right]$ :

$$
U_{m n}(t)=\int_{0}^{\infty} f_{m}^{*} e^{\left(L-\beta_{0}\right) t} g_{n} d x=e^{\left(\beta_{n}-\beta_{0}\right) t} \delta_{m n} .
$$

As only the element with $m=n=0$ survives for large times, the maximal transient amplification is achieved for the "optimal" initial disturbances equal to multiples of $f_{0}$. The evolution amplifies these disturbances and transforms them into multiples of the leading eigenfunction $g_{0}$. Exponential growth with rates predicted by the linear stability analysis sets in rather quickly as the time scales of the exponential and transient growth are of the same order of magnitude (roughly between 2 and 9 nondimensional units, depending on the wavenumber).

The ultimate test of the importance of nonlinear terms and transient dynamics is provided by a direct calculation of the transient amplification factor 


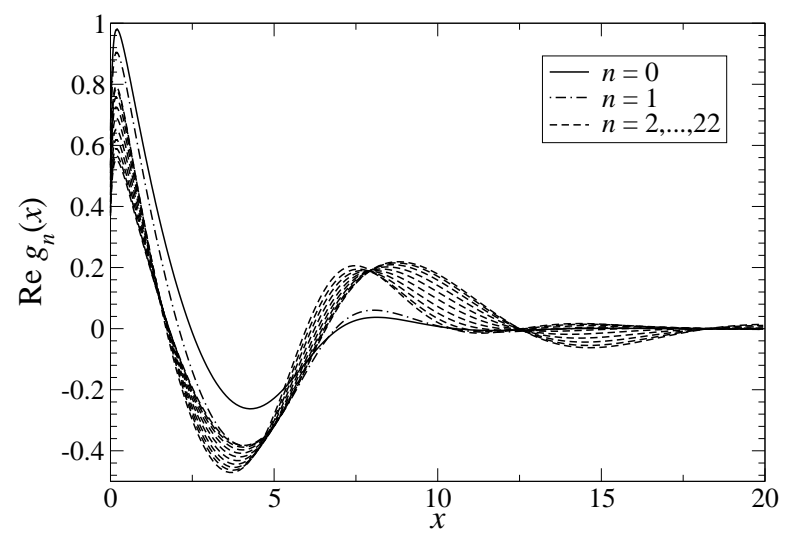

FIG. 8. The eigenfunctions $g_{0}(x)$ through $g_{21}(x)$ for $q=0$, $\alpha=0.01$ and $c=1$. Only a portion of these eigenfunctions, computed on the interval of length $l_{x}=80$, is shown. The eigenfunctions are normalized such that $\int_{0}^{\infty}\left|g_{n}\right|^{2} d x=1$.

$$
\gamma(q)=\max _{t} \gamma(q, t)
$$

The numerical results are presented in Fig. 7. For a fixed contact angle, $\gamma(q)$ is the largest for zero wavenumber disturbances (which do not lead to distortion of the contact line) and quickly decreases with increasing $q$. The maximum transient amplification increases with the contact angle and can become quite significant for typical experimental parameters $(\gamma(0) \approx 70$ for $c=1, \gamma(0) \approx 100$ for $c=1.95)$. However, the effective value for a finite system will likely be in the range of a few tens. The strong transient amplification at $q=0$ can be easily traced to a very close alignment of a large group of eigenfunctions $g_{2}$ through $g_{21}$. As Fig. 8 shows, their shapes are extremely similar. It is, therefore, appropriate to associate the transient behavior with a whole group of stable eigenfunctions. The size of the group increases with $c$, increasing the degree of nonnormality. In contrast, for channel flows apparently only a couple of near-marginal eigenfunctions become closely aligned ${ }^{21}$.

The transient amplification at small $q$ was found to depend rather sensitively on the length of the domain used in the numerical calculations. For instance, the values of $\gamma$ obtained for $l_{x}=30$ were generally about a half of those obtained for $l_{x}=80$. At about $l_{x}=80$ the dependence levelled off, and further increase in $l_{x}$ resulted in large fluctuations in the eigenvalues due to numerical inaccuracies resulting from strong nonnormality of the matrix produced by discretizing $L(q)$. This sensitivity can be explained by the shape of the adjoint eigenfunctions. While the eigenfunctions $g_{n}(x)$ decay exponentially for large $x$, their adjoints $f_{n}(x)$ grow exponentially (see Fig. 9). As a result, small inaccuracies in the boundary conditions at the tail of the film introduced by truncating the computational domain can have a significant effect on the adjoint eigenfunctions, thus affecting the transient amplification, which depends on both $g_{n}(x)$ and $f_{n}(x)$.

The minimal noise level required to trigger the nonlin-

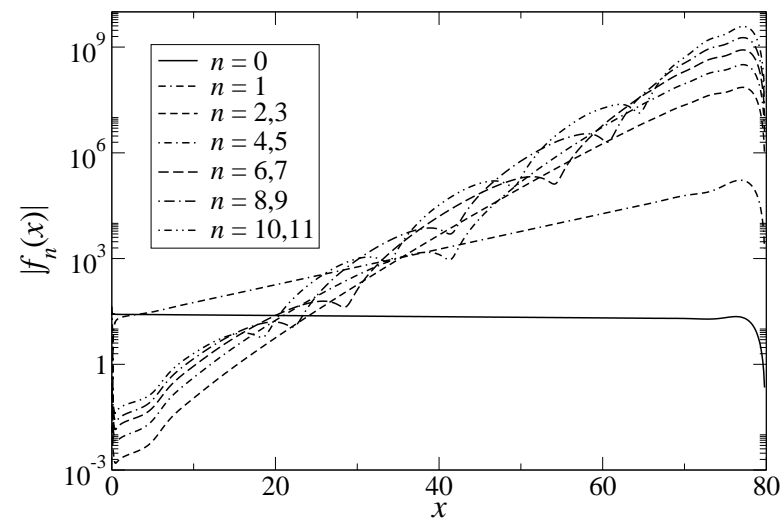

FIG. 9. The adjoint eigenfunctions $f_{0}(x)$ through $f_{11}(x)$ for $q=0, \alpha=0.01$ and $c=1$. The leading eigenfunction $f_{0}(x)$ is constant, aside from a small region near $x=l_{x}$, where it adjusts to the Dirichlet boundary condition. The eigenfunctions are normalized such that $\int_{0}^{\infty} f_{n}^{*} g_{m} d x=\delta_{n m}$.

ear instability according to the second scenario is about $0.1 \%$ of the total film thickness for $\gamma=100$ (the first scenario requires an even higher level of noise). A more accurate calculation of the noise threshold is likely to raise this level much higher (to maybe a few percent) by taking into account the fact that (26) determines the maximal amplification achieved for a specially chosen initial condition, while the secondary disturbances will generically be amplified less strongly. In typical experimental conditions the noise is likely to be substantially smaller than one percent of the film thickness. The existing experimental data ${ }^{4,20}$ agrees with the predictions of the linear theory rather well, supporting the conclusion that the transient effects are relatively weak and, therefore, the modal linear stability analysis of the previous section accurately describes the dynamics.

\section{FEEDBACK CONTROL OF THE CONTACT LINE INSTABILITY}

Now that we understand the limits of the linear stability analysis let us consider the control problem. Can the contact line instability be suppressed, or alternatively, can a pattern with a desired wavelength be imposed by applying feedback? In principle, the answer seems to be clear, as feasibility of feedback control of several other types of instability (buoyancy ${ }^{6}$, thermocapillary ${ }^{7}$, evaporative $^{8}$ ) in liquid layers has been demonstrated. Although one might hope that the developed control methods could be adapted for suppressing the contact line instability, in reality the spreading films turn out to be dramatically different.

The existing control methods have been developed for stabilizing flat films with no mean flow, i.e., steady, uniform target states. The evolution operators describing the dynamics of small disturbances about such states 
have eigenfunctions whose horizontal dependence is given by Fourier modes, which are normal to each other for any realistic boundary conditions (in principle, one can design the boundary conditions such that the eigenfunctions will not be normal even in this case $\left.{ }^{24}\right)$. Not only does it mean that all horizontal modes become uncoupled, so any mode can be controlled independently of the others, there is no nonnormality, so there are no transients and the linear stability analysis is unconditionally valid.

The target state $h_{0}(x+u t)$ in the present problem is nonuniform in the direction of the flow. As a result, the differential operator $L$ does not fully diagonalize and the control problem becomes vastly more complicated. Not only are all the modes in the system coupled, feedback applied to suppress one mode generally affects all other modes, so an infinite-dimensional problem has to be considered from the outset. However, even if these problems are resolved and a feedback making the dynamics asymptotically stable is found, there is no guarantee that the transient effects will not invalidate the whole analysis.

Let us repeat the linear stability analysis of the spreading film, but now in the presence of feedback. First we make use of the simplification afforded by the uniformity of the target state in the transverse direction, which allows us to partially diagonalize the evolution operator. For the moment we restrict our attention to monochromatic disturbances $\epsilon g(x+u t, t) \exp (i q y)$. Since the flow is driven by the gradient in the temperature (and hence surface tension), the stability of the flow is most easily altered by varying the temperature field behind the contact line. Suppose we modify the temperature profile by adding a perturbation

$$
\Delta \theta(x, y, t)=-\epsilon \tau\left(\partial_{\theta} \sigma\right)^{-1} s(t) w(x+u t) e^{i q y},
$$

where the transverse wavelength $q$ is the same as that of the disturbance and $s(t)$ and $w(x)$ are some functions determining the temporal and spatial profile of the perturbation, which will be determined later. Consequently, (7) and hence (14) will be modified to account for the variation in the surface tension transversely to, as well as along, the direction of the flow. At order $\epsilon$ instead of (14) we obtain

$$
\begin{aligned}
\partial_{t} g & =L_{0} g+s\left[N_{1}\left(h_{0}\right) w+N_{2}\left(h_{0}\right) w^{\prime}\right]^{\prime} \\
& +q^{2}\left[L_{1} g-s N_{2}\left(h_{0}\right) w\right]+q^{4} L_{2} g,
\end{aligned}
$$

where the influence functions

$$
\begin{aligned}
& N_{1}\left(h_{0}\right)=\frac{2}{3}\left(h_{0}^{2}-h_{0}\right), \\
& N_{2}\left(h_{0}\right)=h_{0}^{2}+\frac{2}{3}\left(\alpha h_{0}+h_{0}^{3}\right) h_{0}^{\prime \prime}
\end{aligned}
$$

determine the effect of the imposed thermal perturbation.

To get a sense of the dynamics of different modes in the presence of feedback, we expand the disturbance in the basis formed by the eigenfunctions of $L_{0}$,

$$
g(x, t)=\sum_{m} G_{m}(t) g_{m}(x),
$$

and make the strength of the applied perturbation proportional to the magnitude of the distortion of the contact line (with a proportionality constant $k$, called the gain, to be determined later),

$$
s(t)=k \frac{g(0, t)}{c}=\frac{k}{c} \sum_{m} G_{m}(t) g_{m}(0) .
$$

Multiplying (30) by $f_{n}^{*}$ and integrating from 0 to $\infty$ we obtain an infinite system of ODEs describing the dynamics of individual modes:

$$
\dot{G}_{n}=\beta_{n}^{0} G_{n}+\sum_{m}\left(A_{n m}+q^{2} B_{n m}+q^{4} C_{n m}\right) G_{m},
$$

where (assuming that all adjoint eigenfunctions are normalized such that $\left.\int_{0}^{\infty} f_{n}^{*} g_{m} d x=\delta_{n m}\right)$

$$
\begin{aligned}
& A_{n m}=k \frac{g_{m}(0)}{c} \int_{0}^{\infty} f_{n}^{*}\left[N_{1}\left(h_{0}\right) w+N_{2}\left(h_{0}\right) w^{\prime}\right]^{\prime} d x \\
& B_{n m}=\int_{0}^{\infty} f_{n}^{*} L_{1} g_{m} d x-k \frac{g_{m}(0)}{c} \int_{0}^{\infty} f_{n}^{*} N_{2}\left(h_{0}\right) w d x \\
& C_{n m}=\int_{0}^{\infty} f_{n}^{*} L_{2} g_{m} d x .
\end{aligned}
$$

As Fig. 5 shows, the uncontrolled system possesses a single unstable eigenvalue well separated from the rest of the spectrum. One can, therefore, expect that the dynamics of small disturbances should be well described by a single mode truncation of (34) in the absence of feedback. The same is not generally true when the feedback is applied. As all modes are coupled, the feedback designed to suppress the leading mode will always affect, and can potentially destabilize, the subleading modes. As our numerical calculations show, such destabilization does indeed occur, unless the spatial profile $w(x)$ of the thermal perturbation is chosen carefully to avoid this. Had the evolution operator been normal, we could have always chosen the feedback in such a way that different modes became uncoupled, so only the stability of a few independent unstable modes had to be considered. The problem of controlling the contact line instability turns out to be quite delicate in comparison.

Assuming that $w(x)$ is chosen such that all subleading modes remain stable, we can truncate the system (34) by discarding all modes except the leading one. The stability of the single-mode truncation

$$
\dot{G}_{0}=\left(A_{00}+q^{2} B_{00}+q^{4} C_{00}\right) G_{0}
$$

is a necessary condition for the stability of the full system (34). The truncated system is stable when the following three conditions are satisfied:

$$
\begin{aligned}
& A_{00}=k w^{\prime}(\infty) \leq 0 \\
& B_{00}=\int_{0}^{\infty} h_{0}\left(h_{0}-1\right) d x-k \int_{0}^{\infty} N_{2}\left(h_{0}\right) w d x \leq 0 \\
& C_{00}=-\frac{1+2 \alpha}{4} \leq 0
\end{aligned}
$$




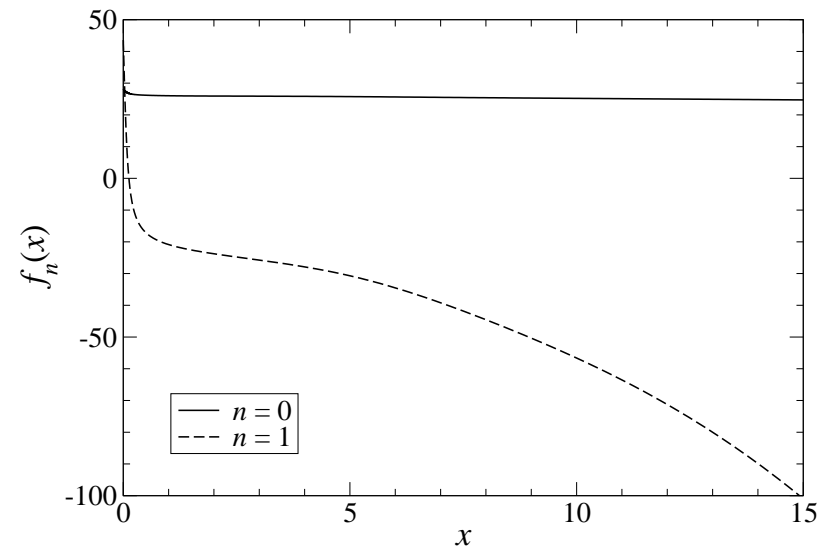

FIG. 10. The adjoint eigenfunctions $f_{0}(x)$ and $f_{1}(x)$ for $q=0, \alpha=0.01$ and $c=1$. Qualitatively similar profiles are obtained for other values of the contact angle. A portion of the eigenvalues computed on the interval of length $l_{x}=80$ is shown.

The first condition is satisfied for any feedback localized near the contact line, because in this case $A_{00}=0$, while the second condition can always be satisfied with the proper choice of the gain $k$. The third condition is clearly satisfied as well. Since (34) is valid for all values of $q$ for which the governing equation (9) is valid and the choice of $k$ in (37) is independent of $q$, we can immediately generalize to non-monochromatic disturbances by integrating over all $q$, such that the feedback will be given by

$$
\Delta \theta(x, y, t)=-k \tau\left(\partial_{\theta} \sigma\right)^{-1} w(x+u t) \xi(y, t),
$$

where $\xi(y, t)$ is the instantaneous deviation of the contact line from its mean position.

How can we choose the heating profile in the flow direction which will stabilize the unstable mode without destabilizing any of the initially stable modes? As Fig. 8 shows, the leading mode is localized under the capillary ridge. Therefore, to suppress the instability one has to apply a perturbation which will be similarly localized to within the region occupied by the ridge. The inspection of matrix elements (35) shows that such a localized perturbation will affect all modes whose adjoint eigenfunctions are not small in that region. According to Fig. 9 both $f_{0}$ and $f_{1}$ are of the same order of magnitude there, while the other subleading modes are several orders of magnitude smaller. As a result, only the stability of the leading and the first subleading mode may change in response to feedback. The numerically computed spectra of the controlled system support this conclusion.

The stability of the two leading modes is determined by the signs and magnitudes of the matrix elements $A_{n m}$ and $B_{n m}$ with $n, m=0,1$. A few general comments about these matrix elements can be made based on the structure of the eigenfunctions. As Fig. 10 shows, $f_{0}$ and $f_{1}$ are both nearly constant under the ridge and have opposite signs, while $g_{0}(0)$ and $g_{1}(0)$ have the same sign.
As a result, a decrease in $B_{00}$ is necessarily accompanied by a commensurate increase in $B_{11}$. Furthermore, since $f_{0}$ is constant, we have $A_{0 n}=0$ for any $n$, so at $q=0$ the eigenvalues of the controlled system are $\beta_{0}^{\prime}(0)=0$ and $\beta_{1}^{\prime}(0) \approx \beta_{1}(0)+A_{11}$. The matrix element $A_{11}$ is generally nonzero and changes in response to the strength of the applied feedback. An inappropriate choice of the profile $w(x)$ or the gain constant $k$ can make $A_{11}$ large enough to cause destabilization at long wavelengths. However, even when $w(x)$ is chosen such that $A_{11}$ is negative (but small), destabilization of the $n=1$ mode at short wavelenghts can occur due to the increase in $B_{11}$, if the feedback gain is too large.

Additional insights can be gained by considering the effect of feedback from the physical point of view. The action of feedback in the direction transverse to the flow is described by the influence function $N_{2}\left(h_{0}\right)$. The first and second term of this function describe the motion of the liquid under the action of, respectively, surface forces and pressure produced by the local gradients in the surface tension. For a convex region of the film, where $h_{0}^{\prime \prime}<0$, these two effects will compete with each other. For instance, a local maximum of surface tension will induce the flow along the surface toward that location and the flow in the interior of the liquid layer away from that location. The first effect will dominate for low capillary ridges (small contact angles), the second one for high capillary ridges (large contact angles).

As the instability is caused by the increased mobility of the capillary ridge, one might envision enforcing control by changing the thickness of the film. The local thickness of the capillary ridge could, in principle, be modified by locally heating or cooling it to redistribute the liquid in such a way as to decrease the thickness, and hence the mobility, where we need to slow down the motion of the contact line and increase the thickness and mobility, where we need to speed it up to compensate for the deviation. This can be achieved by choosing $w(x)$ which is localized under the ridge and does not change sign. For instance, one could pick a Gaussian profile representing the effect of thermal spreading in the solid substrate

$$
w(x)=\exp \left[-\frac{\left(x-x_{0}\right)^{2}}{2 \Delta x^{2}}\right],
$$

where $x_{0}$ and $\Delta x$ are chosen such that $w(x)$ is centered under the capillary ridge and has a comparable width. A sample profile is shown in Fig. 11(a) for a special choice of parameters. The numerically computed spectrum, Fig. 11(b), demonstrates that the stabilization can indeed be achieved by this method for driven films with a relatively low capillary ridge.

This simple approach, however, does not always achieve the desired result. In fact, it only succeeds when the largest growth rate $\beta_{0}\left(q_{\max }\right)$ in the uncontrolled system is small compared to $\left|\beta_{1}(0)\right|$, i.e., when the feedback required to stabilize the $n=0$ mode is too weak to destabilize the $n=1$ mode. One can already notice the sign of 
a)
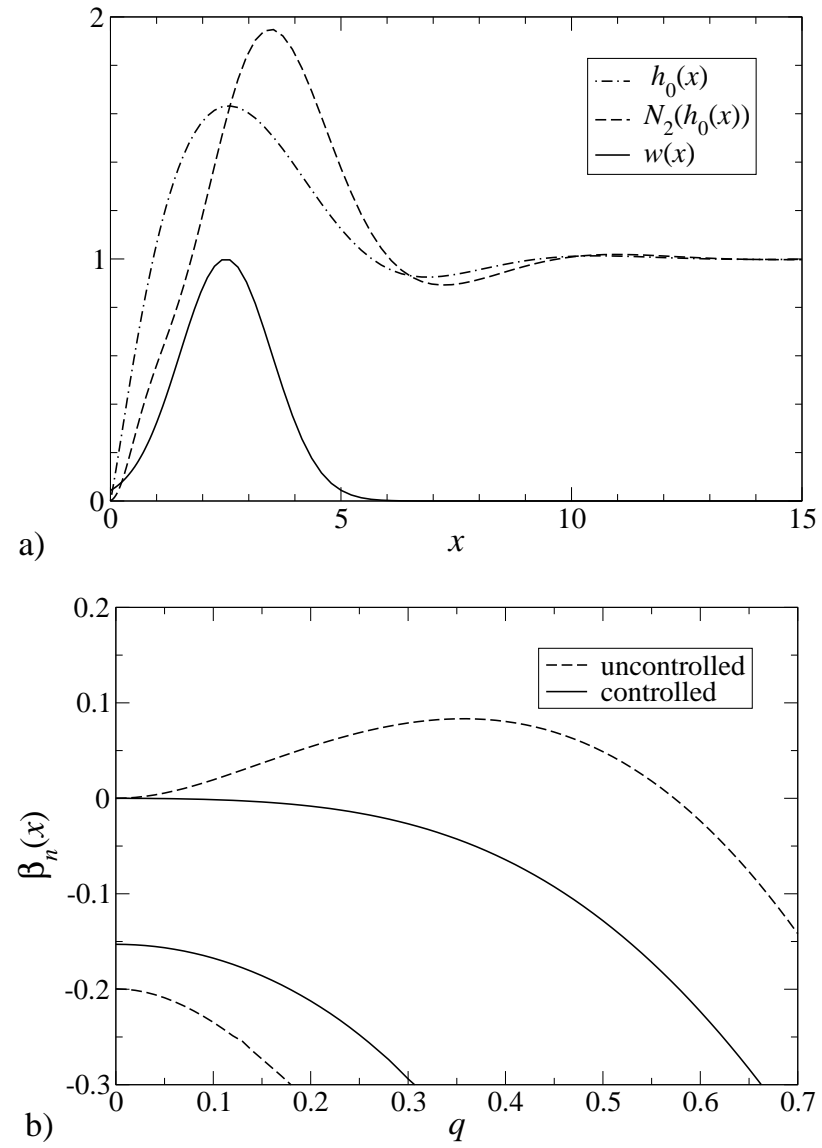

FIG. 11. Stabilization of a thermally driven film with a low capillary ridge, $h_{\max }=1.63(\alpha=0.1$ and $c=1)$. (a) The asymptotic state $h_{0}$, influence function $N_{2}\left(h_{0}\right)$, and thermal perturbation profile $w$. (b) The two leading eigenvalues of the original and controlled system for $k=1$.

approaching trouble by looking at Fig. 11(b): the eigenvalue of the $n=1$ mode at $q=0$ starts to creep upward due to the increase in the matrix element $A_{11}$. If this approach is used to stabilize a flow with $\beta_{0}\left(q_{\max }\right)$ comparable to $\left|\beta_{1}(0)\right|$, the feedback required to suppress the $n=0$ mode becomes strong enough to destabilize the $n=1$ mode at low wavenumbers. The numerically computed spectra for higher capillary ridges $\left(h_{\max }>1.7\right)$ show that the low wavenumbers are destabilized before the high wavenumbers are stabilized for any choice of the width $\Delta x$ of the thermal perturbation.

We are thus forced to look for an alternative solution. Changing the overall thickness of the capillary ridge, even locally, is a rather ineffective procedure, especially for small $q$, as the liquid has to be redistributed over large distances in the transverse direction. One could instead apply a local force to the ridge, redistributing the liquid between its front and back. For instance, by heating the front of the ridge and cooling its back one creates the pressure gradient enhancing the flow. Reversing the sign of the applied perturbation impedes the flow, directly affecting the propagation velocity. The corresponding a)
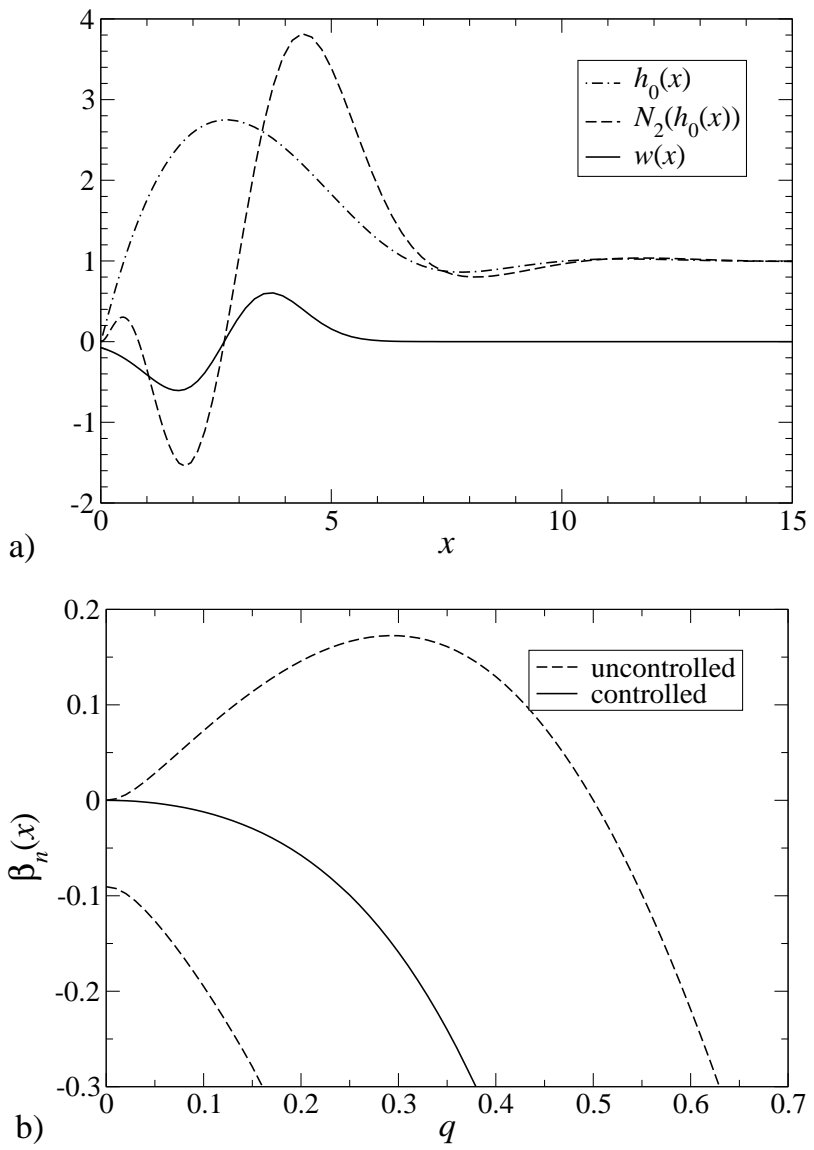

FIG. 12. Stabilization of a thermally driven film with a high capillary ridge, $h_{\max }=2.75(\alpha=0.01$ and $c=1.95)$. (a) The asymptotic state $h_{0}$, influence function $N_{2}\left(h_{0}\right)$, and thermal perturbation profile $w$. (b) The two leading eigenvalues of the original and controlled system for $k=4$. The $n=1$ mode is strongly suppressed by feedback, the respective eigenvalue lies outside the graph.

thermal perturbation should have a profile $w(x)$ which changes the sign near the highest point of the ridge. For instance, if one chooses the profile to be anti-symmetric

$$
w(x)=\left(x-x_{0}\right) \exp \left[-\frac{\left(x-x_{0}\right)^{2}}{2 \Delta x^{2}}\right],
$$

the matrix element $A_{11}$ can be made large and negative, so the $n=0$ mode can be stabilized without destabilizing the $n=1$ mode. Fig. 12(a) shows that such a thermal perturbation with the position $x_{0}$ and width $\Delta x$ tuned to be roughly the same as those of the capillary ridge, should have the largest effect on $B_{00}$. Indeed, the influence function $N_{2}\left(h_{0}\right)$, dominated for high capillary ridges by its second term, also changes sign near the highest point of the capillary ridge. The numerically computed spectrum, Fig. 12(b) shows that one can again successfully suppress the instability.

Another alternative is to exploit the narrow concave region of the film near the contact line. One can again use the Gaussian thermal profile (39) centered at the con- 
a)
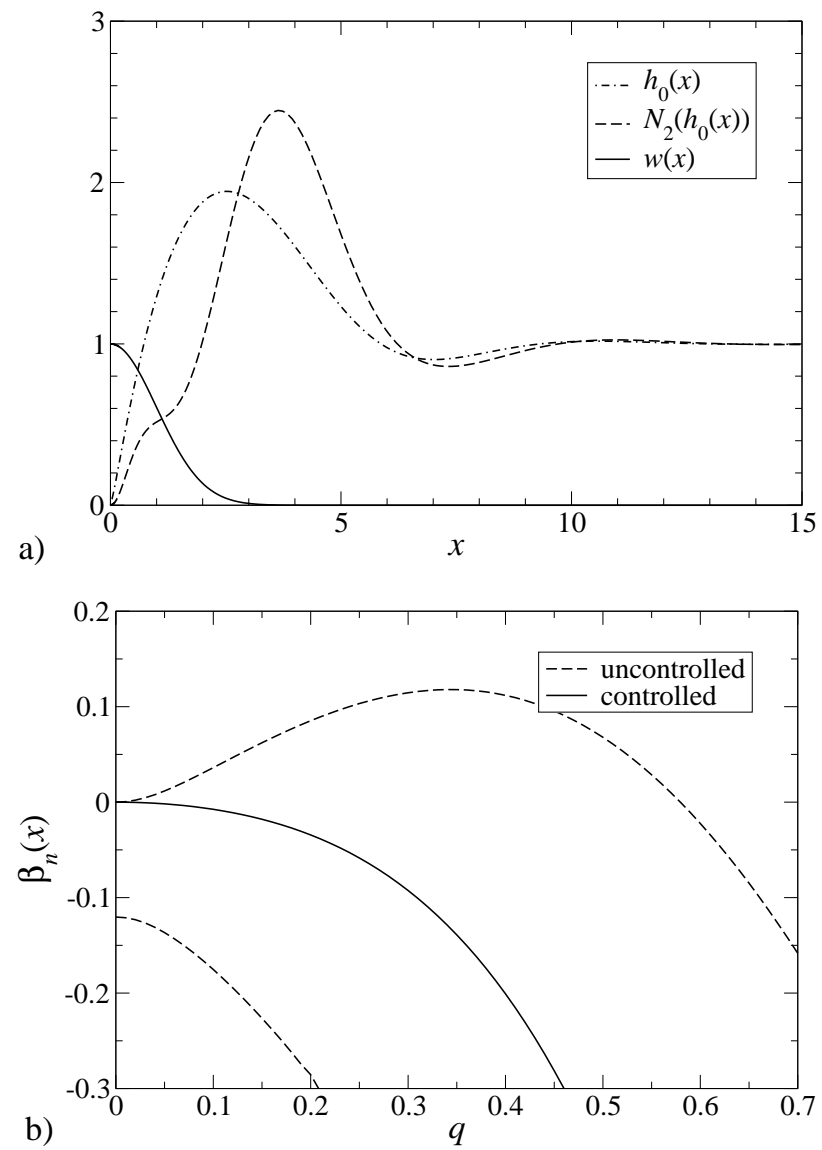

FIG. 13. Stabilization of a thermally driven film with a medium height capillary ridge, $h_{\max }=1.93(\alpha=0.01$ and $c=1)$. (a) The asymptotic state $h_{0}$, influence function $N_{2}\left(h_{0}\right)$, and thermal perturbation profile $w$. (b) The two leading eigenvalues of the original and controlled system for $k=4$. The $n=1$ mode is strongly suppressed by feedback, the respective eigenvalue lies outside the graph.

tact line (see Fig. 13(a)). Heating this region and thus lowering the surface tension one produces gradients in both the pressure and surface tension, which induce the secondary flow away from the contact line. Respectively, cooling this region draws the liquid towards it, providing a direct way to locally control the propagation speed of the film. Making the amount of heating or cooling proportional to the displacement of the contact line again allows one to suppress the contact line instability. The numerically computed spectrum of the system with and without feedback is shown in Fig. 13(b).

Finally, let us look at the transient amplification of disturbances in the presence of feedback. It is unclear $a$ priori what effect the control would have on the transient dynamics. Numerical calculations show that, depending on the choice of the thermal profile $w(x)$, feedback can either increase or decrease the degree of nonnormality in the system. This observation is consistent with the theory. As the matrix elements (35) show, feedback affects different modes in a different way, and small changes in

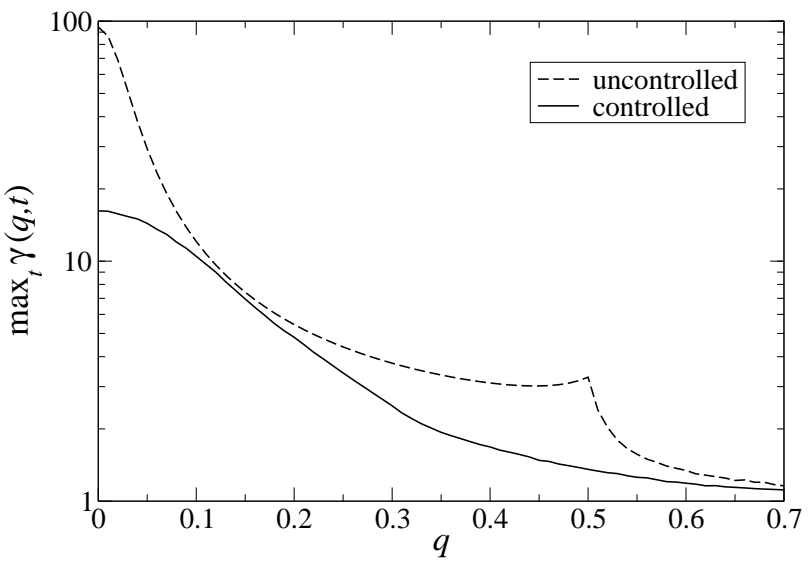

FIG. 14. The transient amplification with and without control for $\alpha=0.01, c=1.95, k=4$, and the anti-symmetric thermal perturbation profile (40).

the eigenvalues can have a large effect on the transient amplification. For instance, thermal perturbations with an anti-symmetric profile, such as (40), can decrease the transient amplification at small wavenumbers by almost an order of magnitude (see Fig. 14). Generally, direct control of the propagation velocity via longitudinal surface tension gradients leads to a decrease in the transient amplification, while indirect control via transverse surface tension gradients affecting the mobility increases the transient amplification for long wavelength disturbances, which is also consistent with naive expectations.

We can thus conclude that an appropriately chosen feedback can make the dynamics asymptotically stable without increasing the transient amplification of disturbances, such that the contact line instability is suppressed in the presence of noise characteristic of typical experimental conditions. Moreover, the feedback is capable of reducing the transient amplification as well, so we can expect that feedback control can be effective in suppressing the contact line instability even when it is caused by the nonlinear effects. Finally, once the instability is suppressed, selective patterning can be achieved by removing feedback and/or introducing additional forcing at a wavenumber corresponding to a desired pattern.

The proposed control algorithm has been verified experimentally. Although no systematic investigation of different thermal perturbation profiles has been attempted so far, the proof-of-principle experiments have shown that by heating the advanced regions of the film and cooling the retarded regions one can completely suppress the contact line instability. It has also been shown that transversely modulated thermal perturbations applied near the contact line can be used to achieve selective patterning, producing perfectly periodic patterns of rivulets. The details of the experiments will be presented in a separate publication (N. Garnier, R. O. Grigoriev, and M. F. Schatz, "Optical manipulation of microscale fluid flow", in preparation). 


\section{DISCUSSION}

To summarize our results, we have determined that the evolution operator governing the dynamics of spontaneous disturbances for thermally driven films, both with and without feedback, is significantly nonnormal and can transiently amplify those disturbances. The strongest transient amplification occurs for the zero wavenumber which does not lead to contact line instability. However, even for nonzero wavenumbers transient amplification is unlikely to produce an instability for levels of noise characteristic of typical experimental conditions. Therefore, linear stability analysis accurately describes both the controlled and the uncontrolled dynamics. In contrast, for gravity driven films at small inclination angles the transient amplification could be much stronger, with the maximum achieved at a nonzero wavenumber ${ }^{12}$, providing an alternative mechanism for instability.

We have also shown that the contact line instability in thermally driven films can be effectively suppressed by locally heating or cooling the liquid behind the contact line. Such thermal perturbation can be easily imposed experimentally with sufficient spatial and temporal resolution by radiatively heating the substrate ${ }^{13}$. This approach offers significant advantages in controlling the dynamics of microflows compared to the one based on chemical patterning of the substrate ${ }^{10,11}$. First of all, no preparation of the substrate is needed, while the patterns can be dynamically reconfigured, offering potential for a significant increase in flexibility. Second, feedback control can be used to achieve extremely small feature size, if high intensity radiation is used to drive the flow on a thin substrate with moderate thermal conductivity ${ }^{8}$, opening up new prospects for microfluidics and microfabrication applications. Finally, feedback control provides a unique opportunity for studying the dynamics of subdominant modes and even unstable states of the system. For instance, it can be used to experimentally measure the growth (or decay) rates of monochromatic disturbances with wavenumbers $q_{0} \neq q_{\max }$ by using a wavenumber dependent gain to suppress the disturbances which would otherwise obscure the dynamics of the mode of interest.

We also expect that feedback control can be equally effective in suppressing nonlinear instabilities such as those occurring in gravity driven spreading at small inclination angles. Indeed, we have seen that the profile of the thermal perturbations can be tuned to decrease the degree of nonnormality. Therefore, by suppressing the transient growth feedback can also quench the bootstrapping mechanism leading to a nonlinear instability. However, because small disturbances at the contact line could be transiently amplified to produce $O(1)$ changes in the thickness of the capillary ridge ${ }^{12}$, it is possible that the control algorithm will have to use direct measurements of the thickness rather than the much easier to monitor position of the contact line.
${ }^{1}$ J. M. Jarrett and J. R. de Bruyn, "Fingering instability of a gravitationally driven contact line," Phys. Fluids A 4, 234 (1992).

2 S. M. Troian, E. Herbolzheimer, S. A. Safran, and J. F. Joanny, "Fingering instabilities of driven spreading films," Europhys. Lett. 10, 25 (1989).

${ }^{3}$ F. Melo, J. F. Joanny, and S. Fauve, "Fingering instability of spinning drops," Phys. Rev. Lett. 63, 1958 (1989).

${ }^{4}$ A. M. Cazabat, F. Heslot, S. M. Troian, and P. Carles, "Fingering instability of thin spreading films driven by temperature gradients," Nature 346, 824 (1990).

${ }^{5}$ D. E. Kataoka and S. M. Troian, "A theoretical study of instabilities at the advancing front of thermally driven coating films," J. Colloid Interface Sci. 192, 350 (1997).

${ }^{6}$ J. Tang, H. H. Bau, "Stabilization of the no-motion state in Rayleigh-Bénard convection through the use of feedbackcontrol," Phys. Rev. Lett. 70, 1795 (1993).

${ }^{7}$ A. C. Or, R. E. Kelly, L. Cortelezzi, and J. L. Speyer, "Control of long-wavelength Marangoni-Bénard convection," J. Fluid Mech. 387, 321 (1999).

${ }^{8}$ R. O. Grigoriev, "Control of evaporatively driven instabilities of thin liquid films," Phys. Fluids 14, 1895 (2002).

${ }^{9}$ D. E. Kataoka and S. M. Troian, "Stabilizing the advancing front of thermally driven climbing films," J. Colloid Interface Sci. 203, 335 (1998).

${ }^{10}$ D. E. Kataoka and S. M. Troian, "Patterning liquid flow on the microscopic scale," Nature 402, 794 (1999).

${ }^{11}$ L. Kondic and J. Diez, "Flow of thin films on patterned surfaces: Controlling the instability," Phys. Rev. E 65, 045301 (2002).

12 A. L. Bertozzi and M. P. Brenner, "Linear stability and transient growth in driven contact lines," Phys. Fluids $\mathbf{9}$, 530 (1997).

${ }^{13}$ D. Semwogerere and M. F. Schatz, "Evolution of hexagonal patterns from controlled initial conditions in a BénardMarangoni convection experiment," Phys. Rev. Lett. 88, 54501 (2002).

${ }^{14}$ A. Oron, S. H. Davis, and S. G. Bankoff, "Long-scale evolution of thin liquid films," Rev. Mod. Phys. 69, 931 (1997).

${ }^{15}$ Dussan V, S. H. Davis, "On the motion of fluid-fluid interface along a solid surface," J. Fluid Mech. 65, 71 (1974).

${ }^{16} \mathrm{H}$. Greenspan, "On the motion of a small viscous droplet that wets a surface," J. Fluid Mech. 84, 125 (1978).

${ }^{17}$ M. A. Spaid and G. M. Homsy, "Stability of Newtonian and viscoelastic dynamic contact lines," Phys. Fluids 8 , 460 (1996).

18 S. Kalliadasis, "Nonlinear instability of a contact line driven by gravity," J. Fluid Mech. 413, 355 (2000).

${ }^{19}$ E. O. Tuck and L. W. Schwartz, "A numerical and asymptotic study of some third-order ordinary differential equations relevant to draining and coating flows," SIAM Rev. 32, 453 (1990).

${ }^{20}$ J. B. Brzoska, F. Brochard-Wyart, and F. Rondelez, "Exponential-growth of fingering instabilities of spreading films under horizontal thermal-gradients," Europhys. Lett. 
19, 97 (1992).

${ }^{21}$ L. N. Trefethen, A. E. Trefethen, S. C. Reddy, and T. A. Driscoll, "Hydrodynamic stability without eigenvalues," Science 261, 578 (1993).

${ }^{22} \mathrm{Y}$. Ye and H.-C. Chang, "A spectral theory for fingering on a prewetted plane," Phys. Fluids 11, 2494 (1999).

${ }^{23}$ B. F. Farrell and P. J. Ioannou, "Generalized stability theory part I: autonomous operators," J. Atmospheric Sci. 53, 2025 (1996)

${ }^{24}$ R. O. Grigoriev and A. Handel, "Nonnormality and the localized control of extended systems," to be published by Phys. Rev. E. 